\title{
201 BNT221, AN AUTOLOGOUS NEOANTIGEN-SPECIFIC T- CELL PRODUCT FOR ADOPTIVE CELL THERAPY OF METASTATIC OVARIAN CANCER
}

${ }^{1}$ Christina Arieta*, ${ }^{1}$ Diana Velez, ${ }^{1}$ Susan Hannes, 'Shirisha Meda, ${ }^{1}$ Brian McCarthy,


${ }^{1}$ Daniel Kallin, ${ }^{1}$ Paul Turcott, ${ }^{2}$ Cynthia Nijenhuis, ${ }^{2}$ Maarje Rohaan, ${ }^{2}$ Gabe Sonke, ${ }^{2} J$ ohn Haanen, ${ }^{1}$ Mark DeMario, ${ }^{1}$ Richard Gaynor, 'Marit Van Buuren. ${ }^{1}$ BioNTech US, Cambridge, USA; ${ }^{2}$ Netherlands Cancer Institute, Amsterdam, Netherlands

Background Neoantigens are tumor-specific antigens that are important in the anti-tumor immune response. These antigens are not subject to central immune tolerance and are therefore potentially more immunogenic than tumor-associated antigens. Here, we present the results of a proof-of-concept, pre-clinical study with multiple successful patient material runs generating a neoantigen-specific T-cell product (BNT221/NEO-PTC-01) using leukaphereses from patients with ovarian cancer. These products contain specific $\mathrm{T}$-cell responses targeting multiple neoantigens from each individual patient's tumor.

Methods Leukapheresis and tumor biopsy samples were obtained from multiple patients with ovarian cancer and metastatic melanoma cancer under IRB approval using the N16NEON protocol at the Netherlands Cancer Institute subsidized by BioNTech US. Patient-specific neoantigens from the patient's biopsy were predicted using our RECON ${ }^{\circledR}$ bioinformatics platform and the best scoring neoantigens were encoded into synthetic peptides or mRNA molecules and were utilized in our ex vivo stimulation protocol, NEO-STIM ${ }^{\circledR}$, which is used to prime, activate, and expand memory and de novo T-cell responses from both the CD4+ and the CD8+ compartment. High-throughput flow cytometric analysis was performed to characterize the specificity and functionality (cytokine production and cytolytic capacity) of the induced Tcell responses.

Results NEO-STIM generates T-cell products specific to neoantigens from the peripheral blood of patients. Data will be presented showing the successful induction of 2-10 CD8+ and 4-13 CD4+ T-cell responses per patient, generated using peripheral blood mononuclear cells from patients with ovarian cancer using our NEO-STIM platform. We extensively characterized these T-cell responses and demonstrate that these responses are polyfunctional, specific and have the capacity to degranulate. $\mathrm{T}$ cells in the induced product are of effector memory and central memory phenotypes.

Conclusions NEO-STIM is a novel platform that generates ex vivo T-cell responses to high-quality neoantigen targets. Efforts are ongoing to upscale the manufacturing process and move this into a phase I study. BNT221, the neoantigen-specific T cell product generated from this process, is a potent adoptive cell therapy targeting multiple immunogenic neoantigens in patients with metastatic ovarian cancer.

Ethics Approval Leukapheresis and tumor biopsy samples were obtained from multiple patients with ovarian cancer and metastatic melanoma cancer under IRB approval using the N16NEON protocol at the Netherlands Cancer Institute subsidized by BioNTech US.

http://dx.doi.org/10.1136/jitc-2021-SITC2021.201 